\title{
Management of Home Blood Pressure by Amlodipine Combined With Angiotensin II Receptor Blocker in Type 2 Diabetes
}

\author{
Katsumi Miyauchi, MD; Tsutomu Yamazaki, MD, PhD; Hirotaka Watada, MD, PhD; \\ Yasushi Tanaka, MD, PhD; Ryuzo Kawamori, MD, PhD; Yutaka Imai, MD, PhD; \\ Shunya Ikeda, MD, PhD; Akira Kitagawa; Yasuhiro Ono, MD, PhD; \\ Fumio Murayama, MD, PhD; Jong Bock Choi, MD, PhD; Satoru Suwa, MD; \\ Dobun Hayashi, MD, PhD; Junji Kishimoto, PhD; Hiroyuki Daida, MD \\ for the ADVANCED-J investigators
}

\begin{abstract}
Background: Angiotensin II receptor blocker (ARB) as a first-line drug for hypertension in diabetes often fails to control blood pressure adequately. The objective of the study was to evaluate the effect of amlodipine combined therapy on home blood pressure (HBP) useful for management of hypertension.

Methods and Results: A total of 263 type 2 diabetes with hypertension refractory to standard dose of ARB were randomized to increased $A R B$ regimen $(n=132)$ or amlodipine combination regimen $(n=131)$. The primary endpoint was change in morning HBP at 1 year. The combination regimen significantly lowered morning HBP than the increased ARB regimen (158.2/82.5 $\mathrm{mmHg}$ in the combination regimen, $157.3 / 84.4 \mathrm{mmHg}$ in the increased ARB regimen, at baseline; $142.7 / 76.3$ vs. $155.0 / 83.1 \mathrm{mmHg}$, respectively, $\mathrm{P}<0.001$ for both, at 8 weeks; $139.6 / 74.6$ vs. $149.1 / 78.1 \mathrm{mmHg}$, respectively, $\mathrm{P}<0.001$ for systolic and $\mathrm{P}=0.010$ for diastolic, at 1year). The combination regimen showed significantly higher rates of achieving target morning HBP at 8 weeks $(11.3 \%$ vs. $2.7 \%, P=0.015)$. In the combination regimen, estimated glomerular filtration rate declined slower, and carotid intima-media thickness decreased in contrast to the increased ARB regimen.
\end{abstract}

Conclusions: In type 2 diabetes patients with hypertension refractory to standard dose of ARB, the amlodipine combination regimen provides superior antihypertensive effect on HBP to the increased ARB regimen, and beneficial effects on reducing risks of cardiovascular events. (Circ $J$ 2012; 76: 2159-2166)

Key Words: Angiotensin II receptor blocker; Calcium channel blocker; Home blood pressure; Hypertension; Type 2 diabetes

D iabetes mellitus comorbid with hypertension increases the incidence of cardiovascular disease..$^{1,2}$ These patients benefit considerably from the achievement of target blood pressure levels below those generally recommended for hypertensive patients, ${ }^{3}$ and guideline levels are lower than those for patients with hypertension alone. Although adequate control of blood pressure in these patients produces a dramatic decrease in the incidence of cardiovascular and cerebrovascular events, ${ }^{4-6}$ few studies have attempted to optimize treatment in those refractory to standard antihypertensive care.

Received December 2, 2011; revised manuscript received April 17, 2012; accepted April 27, 2012; released online June 9, 2012 Time for primary review: 7 days

Department of Cardiology, Juntendo University School of Medicine, Tokyo (K.M., H.D.); Department of Clinical Epidemiology and Systems, Graduate School of Medicine, The University of Tokyo, Tokyo (T.Y.); Department of Medicine, Metabolism \& Endocrinology, Juntendo University School of Medicine, Tokyo (H.W., J.B.C.); Department of Medicine, Metabolism and Endocrinology, St. Marianna University, School of Medicine, Kawasaki (Y.T.); Sportology Center, Juntendo University Graduate School of Medicine, Tokyo (R.K.); Department of Planning for Drug Development and Clinical Evaluation, Tohoku University Graduate School, Sendai (Y.I.); Department of Pharmaceutical Sciences, International University of Health and Welfare, Otawara (S.I.); Kanazawa Medical Clinic, Yokohama (A.K.); Department of Medicine, Takagi Hospital, Okawa (Y.O.); Department of Respiratory Surgery, International University of Health and Welfare Atami Hospital, Atami (F.M.); Department of Cardiology, Juntendo University Shizuoka Hospital, Shizuoka (S.S.); Healthcare Center, Nippon Yusen Kaisya, Tokyo (D.H.); and Center for Clinical and Translational Research, Kyushu University Hospital, Fukuoka (J.K.), Japan

Mailing address: Katumi Miyauchi, MD, Department of Cardiology, Juntendo University School of Medicine, 2-1-1 Hongo, Bunkyo-ku, Tokyo 113-8421, Japan. E-mail: ktmmy@juntendo.ac.jp

ISSN-1346-9843 doi:10.1253/circj.CJ-11-1406

All rights are reserved to the Japanese Circulation Society. For permissions, please e-mail: cj@j-circ.or.jp 


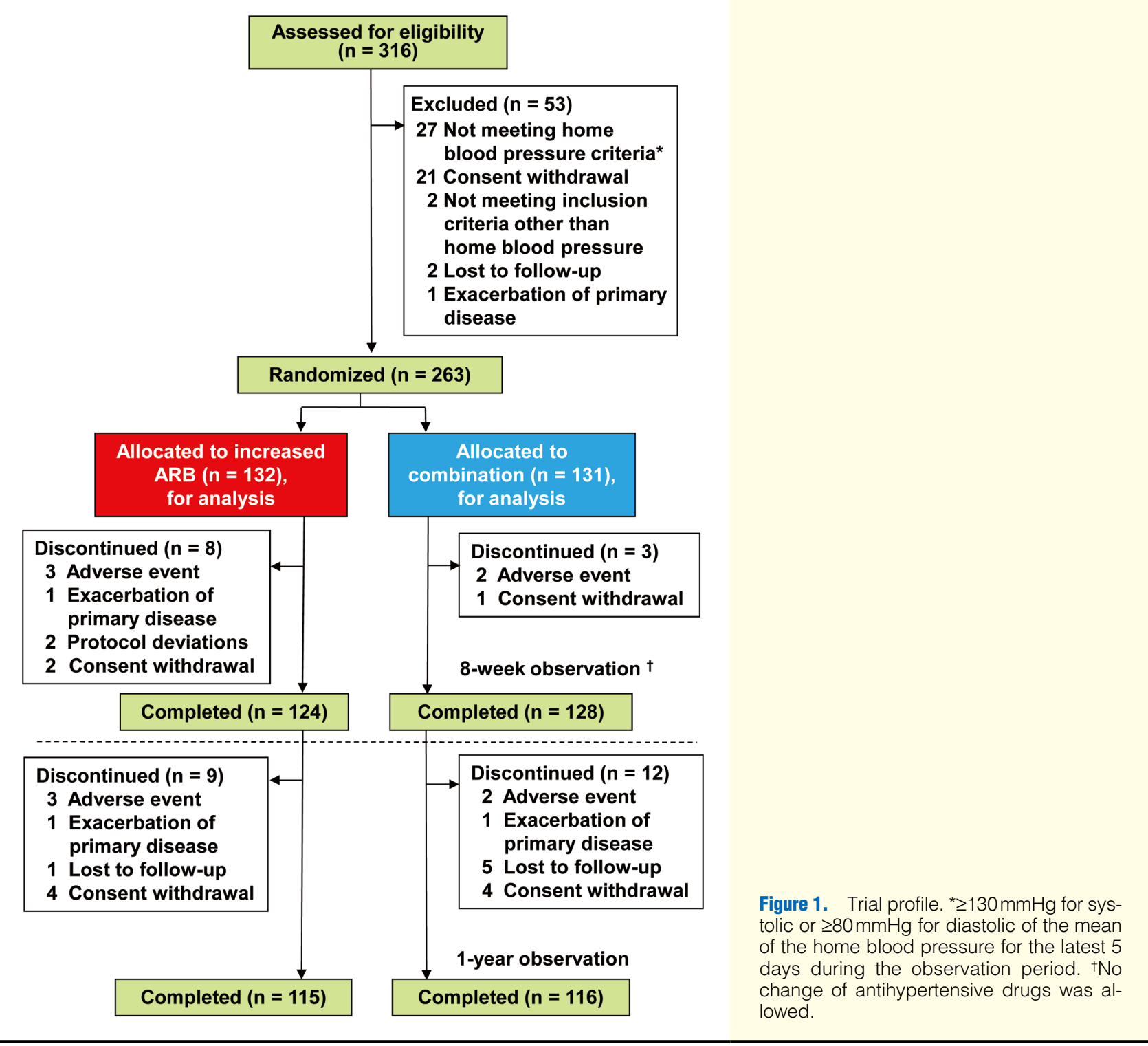

A number of studies have demonstrated that the monitoring only at the clinical visits was insufficient to control blood pressure adequately. Among the results, 1 study identified a discrepancy between screening and ambulatory blood pressure, ${ }^{7}$ while a second showed that the measurement of morning home blood pressure (HBP) has stronger predictive power for mortality than office blood pressure measurement. ${ }^{8}$ Strict control of blood pressure in diabetic patients with hypertension achieves reduction in the risk of deaths, complications related to diabetes, progression of diabetic retinopathy, and deterioration in visual acuity. ${ }^{9}$ These findings both call into question conventional blood pressure monitoring practices and highlight the need for strict hypertensive monitoring in diabetic patients with hypertension.

Thus, we conducted the ADVANCED-J (Amlodipine Versus Angiotensin II Receptor Blocker, Control of Blood Pressure Evaluation Trial in Diabetics) to evaluate the effect of amlodipine combined with the standard dose of angiotensin II receptor blocker (ARB) on HBP for 3 years. We report the 1year follow-up results of the study (UMIN Clinical trial regis- try: C000000017).

\section{Methods}

The ADVANCE-J study is a prospective, multicenter, randomized study to compare the effect of amlodipine combination regimen on HBP with that of increased dose of $\mathrm{ARB}$ for type 2 diabetic patients with hypertension refractory to standard dose of ARB.

\section{Subjects}

The protocol of the ADVANCE-J study has been published elsewhere. ${ }^{10}$ Briefly, eligible patients were aged 20 years or older with type 2 diabetes mellitus and hypertension refractory to standard dose of ARB for at least 8 weeks, whose mean morning home systolic blood pressure was $\geq 130 \mathrm{mmHg}$ or diastolic blood pressure $\geq 80 \mathrm{mmHg}$ for the latest 5 days of the observation period. Patients were excluded when the patient had secondary hypertension, severe hepatic or renal dysfunction, or history of hypersensitivity to the study drugs. The present 


\begin{tabular}{|c|c|c|c|}
\hline & $\begin{array}{c}\text { Increased ARB } \\
(n=132)\end{array}$ & $\begin{array}{c}\text { Combination } \\
(n=131)\end{array}$ & P value* \\
\hline Age (years) & $65.1 \pm 9.4$ & $65.1 \pm 10.0$ & 0.999 \\
\hline Gender, male & $85(64.4)$ & $77(58.8)$ & 0.349 \\
\hline $\mathrm{BMI}\left(\mathrm{kg} / \mathrm{m}^{2}\right)$ & $25.1 \pm 3.4$ & $25.4 \pm 4.1$ & 0.573 \\
\hline Smokers & $27(20.5)$ & $32(24.4)$ & 0.440 \\
\hline Duration of hypertension (years) & $6.7 \pm 7.9$ & $6.8 \pm 7.3$ & 0.884 \\
\hline Duration of diabetes (years) & $6.0 \pm 5.9$ & $8.1 \pm 7.4$ & 0.014 \\
\hline Dyslipidemia & $57(43.2)$ & $62(47.3)$ & 0.499 \\
\hline \multicolumn{4}{|l|}{ History of: } \\
\hline Cerebrovascular disease ${ }^{\dagger}$ & $13(9.9)$ & $11(8.4)$ & 0.683 \\
\hline Cardiovascular disease $e^{\ddagger}$ & $21(15.9)$ & $20(15.3)$ & 0.886 \\
\hline Cardiac failure & $2(1.5)$ & $3(2.3)$ & 0.645 \\
\hline Diabetic nephropathy & $25(18.9)$ & $29(22.1)$ & 0.521 \\
\hline Diabetic retinopathy & $32(24.2)$ & $30(22.9)$ & 0.798 \\
\hline Diabetic neuropathy & $16(12.1)$ & $20(15.3)$ & 0.458 \\
\hline \multicolumn{4}{|l|}{ ARB at baseline: } \\
\hline Candesartan & $41(31.1)$ & $38(29.0)$ & 0.717 \\
\hline Losartan & $16(12.1)$ & $18(13.7)$ & 0.696 \\
\hline Telmisartan & $28(21.2)$ & $27(20.6)$ & 0.905 \\
\hline Valsartan & $39(29.5)$ & $41(31.3)$ & 0.757 \\
\hline Olmesartan & $8(6.1)$ & $7(5.3)$ & 0.802 \\
\hline
\end{tabular}

Quantitative variables were expressed as mean \pm SD.

( ), \%. *Comparability was tested by chi-square test for categorical variables, and by 2-sample t-test for quantitative variables; ${ }^{\dagger}$ cerebral hemorrhage and cerebral infarction; ¥angina pectoris and myocardial infarction.

ARB, angiotensin II receptor blocker; SD, standard deviation.

\begin{tabular}{|c|c|c|c|}
\hline & $\begin{array}{c}\text { Increased ARB } \\
\quad(n=132)\end{array}$ & $\begin{array}{c}\text { Combination } \\
(n=131)\end{array}$ & P value* \\
\hline Not added & $53(40.2)$ & $89(67.9)$ & $<0.001$ \\
\hline Added ${ }^{\dagger}$ & $79(59.8)$ & $42(32.1)$ & \\
\hline \multicolumn{4}{|c|}{ Antihypertensive drugs added ${ }^{\dagger}$} \\
\hline$a$-blocker & $50(37.9)$ & $32(24.4)$ & 0.017 \\
\hline$\beta$-blocker & $31(23.5)$ & $18(13.7)$ & 0.042 \\
\hline Diuretic & $18(13.6)$ & $4(3.1)$ & 0.002 \\
\hline Others ${ }^{\ddagger}$ & $10(7.6)$ & $1(0.8)$ & 0.006 \\
\hline $\mathrm{CCB}$ & $5(3.8)$ & $1 \S(0.8)$ & \\
\hline
\end{tabular}

( ), \%. *Comparability was tested by chi-square test; tincluding drug changed; $¥ a 2$-adrenoreceptor agonist, vasodilator; §a case in which amlodipine was changed to nifedipine.

ARB, angiotensin II receptor blocker; CCB, calcium-channel blocker.

study adhered to the principle of the Declaration of Helsinki. The study protocol was approved by the ethics committee of each participating institute. All patients gave written informed consent prior to participating in the study.

\section{Study Treatment}

Patients eligible for the study were randomly allocated to the increased ARB group or the amlodipine combination group. Study drugs were administrated once in the morning. Patients received the standard dose of $\mathrm{ARB}$ with candesartan $8 \mathrm{mg}$, losartan $50 \mathrm{mg}$, telmisartan $40 \mathrm{mg}$, valsartan $80 \mathrm{mg}$, or olmesartan $20 \mathrm{mg}$ daily during the observation period. In the increased ARB group, dose of ARB increased to the maximal dose approved, $12 \mathrm{mg}, 100 \mathrm{mg}, 80 \mathrm{mg}, 160 \mathrm{mg}$, or $40 \mathrm{mg}$ during the study period, respectively. In the combination group, patients received amlodipine of $5 \mathrm{mg}$ /day combined with the standard dose of ARB.

Antihypertensive drugs used concomitantly with ARB dur- ing the observation period were continued during the study period. In principle, no change of antihypertensive drugs was allowed from allocation throughout the 8-week study period. Diabetes medication was continued in accordance with the guidelines of the Japan Diabetes Society. Patients were advised to adopt positive lifestyle changes, including dietary counseling, smoking cessation, and suitable exercise regimens.

\section{Measurement of HBP}

HBP was self-measured using an automated sphygmomanometer (HEM-705IT, OMRON Healthcare Co, Ltd, Kyoto, Japan) with a data transmitter which transferred data to the central data center automatically. Patients were instructed to measure their blood pressure after 5 min of rest in a sitting position after arising and before going to bed. 


\begin{tabular}{|c|c|c|c|c|c|}
\hline \multirow{2}{*}{ Treatment group } & \multirow{2}{*}{$\begin{array}{c}\text { Baseline } \\
\text { Mean } \pm \text { SD (n) }\end{array}$} & \multicolumn{2}{|c|}{8 weeks } & \multicolumn{2}{|l|}{1 year } \\
\hline & & Mean \pm SD (n) & P value* & Mean \pm SD (n) & P value \\
\hline \multicolumn{6}{|c|}{ Morning home SBP $(\mathrm{mmHg})$} \\
\hline Increased ARB & $157.3 \pm 16.6(132)$ & $155.0 \pm 17.5(112)$ & $<0.001$ & $149.1 \pm 19.9(86)$ & $<0.001$ \\
\hline Combination & $158.2 \pm 19.2(131)$ & $142.7 \pm 15.8(106)$ & & $139.6 \pm 12.2(91)$ & \\
\hline \multicolumn{6}{|c|}{ Morning home DBP $(\mathrm{mmHg})$} \\
\hline Increased ARB & $84.4 \pm 11.6(132)$ & $83.1 \pm 11.4(112)$ & $<0.001$ & $78.1 \pm 12.2(86)$ & 0.010 \\
\hline Combination & $82.5 \pm 11.8(131)$ & $76.3 \pm 9.6(106)$ & & $74.6 \pm 9.5(91)$ & \\
\hline \multicolumn{6}{|c|}{ Evening home SBP $(\mathrm{mmHg})$} \\
\hline Increased ARB & $151.0 \pm 21.0(115)$ & $148.1 \pm 20.7(102)$ & $<0.001$ & $142.6 \pm 17.2(78)$ & $<0.001$ \\
\hline Combination & $149.8 \pm 20.6(121)$ & $134.1 \pm 16.4(102)$ & & $130.8 \pm 14.2(78)$ & \\
\hline \multicolumn{6}{|c|}{ Evening home DBP $(\mathrm{mmHg})$} \\
\hline Increased ARB & $77.5 \pm 11.6(115)$ & $75.4 \pm 11.4(102)$ & $<0.001$ & $72.2 \pm 11.4(78)$ & 0.004 \\
\hline Combination & $74.9 \pm 11.2(121)$ & $69.0 \pm 8.8(102)$ & & $66.3 \pm 8.6(78)$ & \\
\hline \multicolumn{6}{|l|}{ Office SBP $(\mathrm{mmHg})$} \\
\hline Increased ARB & $150.9 \pm 15.5(132)$ & $145.9 \pm 16.0(116)$ & $<0.001$ & $140.9 \pm 17.9(106)$ & $<0.001$ \\
\hline Combination & $152.0 \pm 15.7(131)$ & $134.6 \pm 13.4(114)$ & & $133.8 \pm 11.8(102)$ & \\
\hline \multicolumn{6}{|l|}{ Office DBP $(\mathrm{mmHg})$} \\
\hline Increased ARB & $83.7 \pm 10.3(132)$ & $80.1 \pm 10.5(116)$ & $<0.001$ & $75.8 \pm 12.3(106)$ & 0.207 \\
\hline Combination & $82.7 \pm 11.5(131)$ & $73.7 \pm 11.3(114)$ & & $73.5 \pm 9.4(102)$ & \\
\hline
\end{tabular}

${ }^{*}$ Changes between the 2 treatment groups were assessed using analysis of covariance, including treatment groups as the main effect and BP at baseline as covariate.

BP, blood pressure; SD, standard deviation; SBP, systolic BP; DBP, diastolic BP; ARB, angiotensin II receptor blocker.

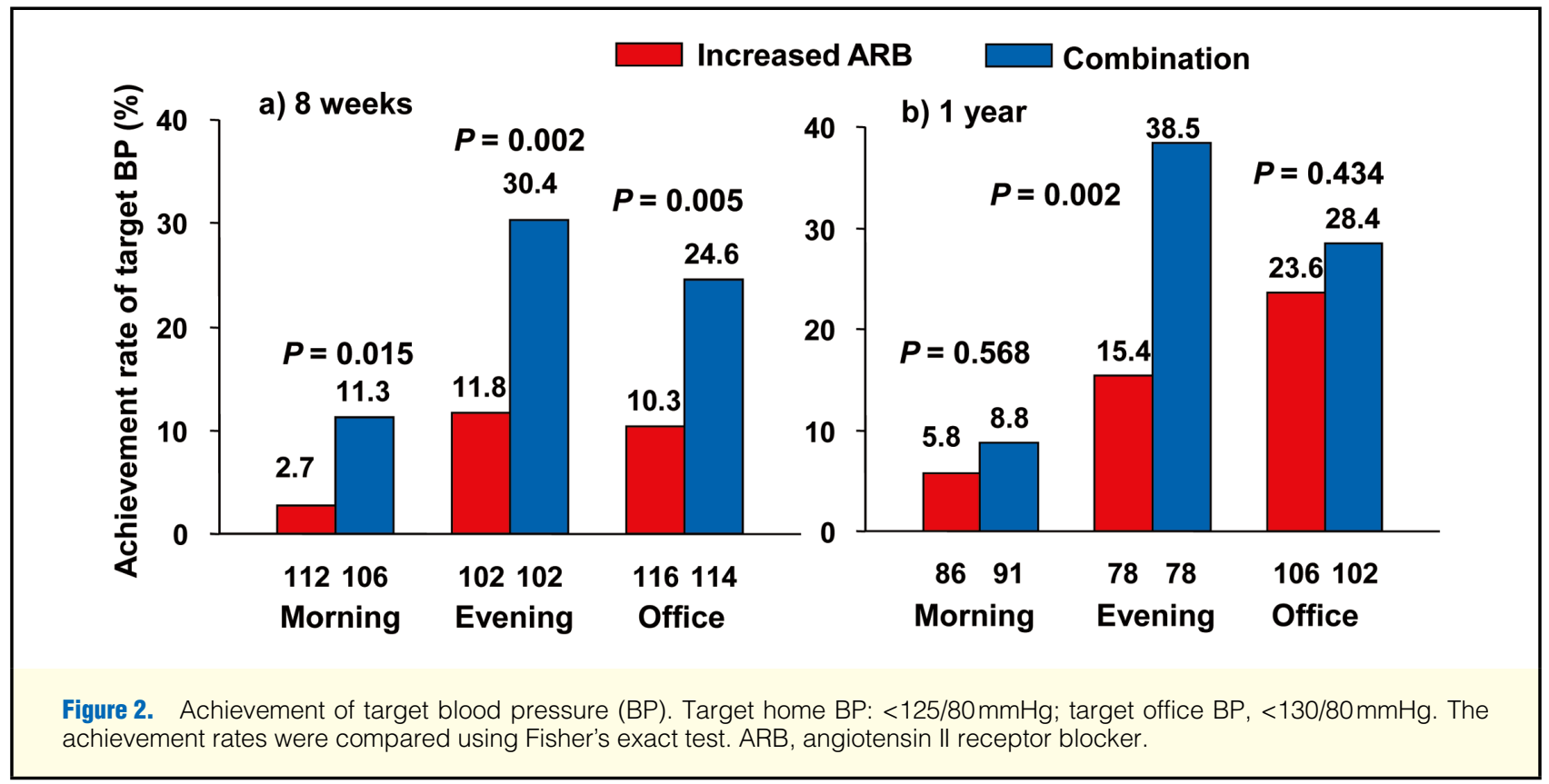

\section{Clinical Endpoints}

The primary endpoint was change in morning HBP at 1 year (Protocol committee amended the protocol on November 18,2007 , before data analysis). Secondary endpoints included changes in morning HBP, evening HBP, and office blood pressure during the study period, and the achievement rates of target blood pressure level (HBP: $<125 / 80 \mathrm{mmHg}$, office blood pressure: $<130 / 80 \mathrm{mmHg}$ ). Other parameters included changes in $\mathrm{HbA}_{1}$, B-type natriuretic peptide (BNP), urinary albumin (calculated as creatinine equivalent), and carotid intima-media thickness (IMT). Carotid IMT was scored using the software, Intima Scope ${ }^{\circledR}$ (SoftMedical Co Ltd, Tokyo, Japan) at the core laboratory of this study, which was organized at the Department of Medicine, Metabolism \& Endocrinology, Juntendo University School of Medicine. The core laboratory provided the average IMT, which was the mean value of 60 computerbased points in the $20 \mathrm{~mm}$ proximal region to the origin of the carotid bulb.

\section{Statistical Analysis}

The target number of patients was estimated to be 300 patients (150 patients in each arm) to detect a difference of $5 \mathrm{mmHg}$ in 


\begin{tabular}{|c|c|c|c|c|c|c|c|}
\hline \multirow{2}{*}{ Treatment group } & \multirow{2}{*}{$\begin{array}{c}\text { Baseline } \\
\text { Mean } \pm \text { SD (n) }\end{array}$} & \multicolumn{3}{|c|}{8 weeks } & \multicolumn{3}{|c|}{1 year } \\
\hline & & Mean \pm SD (n) & P value* & $\mathbf{P}$ value $^{\dagger}$ & Mean \pm SD (n) & P value* & $P$ value \\
\hline \multicolumn{8}{|l|}{$\mathrm{HbA}_{1 \mathrm{c}}(\%)$} \\
\hline ARB & $7.13 \pm 0.93(129)$ & $7.13 \pm 0.99(113)$ & 0.695 & 0.093 & $7.16 \pm 1.16(103)$ & 1.000 & 0.177 \\
\hline Combination & $7.25 \pm 1.02(130)$ & $7.39 \pm 1.08(109)$ & 0.142 & & $7.03 \pm 0.90(103)$ & 0.044 & \\
\hline \multicolumn{8}{|c|}{ eGFR $\left(\mathrm{ml} \cdot \mathrm{min}^{-1} \cdot 1.73 \mathrm{~m}^{-2}\right)$} \\
\hline ARB & $72.34 \pm 19.14$ (129) & $72.23 \pm 20.51(111)$ & 0.494 & 0.832 & $69.22 \pm 19.00(107)$ & 0.005 & 0.163 \\
\hline Combination & $70.82 \pm 18.80(130)$ & $70.44 \pm 19.75$ (112) & 0.556 & & $69.80 \pm 21.85$ (105) & 0.323 & \\
\hline \multicolumn{8}{|l|}{ Average IMT (mm) } \\
\hline ARB & $0.864 \pm 0.189(116)$ & ND & - & - & $0.886 \pm 0.177(75)$ & 0.242 & 0.073 \\
\hline \multirow[t]{2}{*}{ Combination } & $0.879 \pm 0.180(115)$ & ND & - & - & $0.872 \pm 0.177(77)$ & 0.172 & \\
\hline & $\begin{array}{c}\text { Median [mean] } \\
(25-75 \%),(n)\end{array}$ & $\begin{array}{l}\text { Median [mean] } \\
(25-75 \%),(n)\end{array}$ & P value* & P value $^{\dagger}$ & $\begin{array}{c}\text { Median [mean] } \\
(25-75 \%),(n)\end{array}$ & P value* & P value \\
\hline \multicolumn{8}{|l|}{ BNP (pg/ml) } \\
\hline ARB & $\begin{array}{c}25.5[51.7] \\
(12.4-59.3),(117)\end{array}$ & $\begin{array}{c}21.6[59.3] \\
(12.2-81.0),(95)\end{array}$ & 0.570 & 0.062 & $\begin{array}{c}29.8[65.5] \\
(15.0-82.1),(97)\end{array}$ & 0.019 & 0.049 \\
\hline Combination & $\begin{array}{c}26.5[41.0] \\
(13.4-43.2),(122)\end{array}$ & $\begin{array}{c}20.0[35.1] \\
(11.6-39.1),(100)\end{array}$ & 0.002 & & $\begin{array}{c}26.2[45.5] \\
(12.1-57.6),(91)\end{array}$ & 0.847 & \\
\hline \multicolumn{8}{|l|}{ Urinary albumin $(\mathrm{mg} / \mathrm{gCr})$} \\
\hline ARB & $\begin{array}{c}30.1[228.4] \\
(12.4-148.7),(131)\end{array}$ & $\begin{array}{c}29.0[356.0] \\
(11.1-202.0),(93)\end{array}$ & 0.343 & 0.382 & $\begin{array}{c}24.9[226.0] \\
(10.2-133.2),(93)\end{array}$ & 0.253 & 0.714 \\
\hline Combination & $\begin{array}{c}31.8[268.9] \\
(11.9-97.4),(130)\end{array}$ & $\begin{array}{c}33.0[313.7] \\
(11.9-109.7),(104)\end{array}$ & 0.836 & & $\begin{array}{c}22.0[297.3] \\
(9.3-100.2),(90)\end{array}$ & 0.558 & \\
\hline
\end{tabular}

eGFR was estimated using the following equation of the Japanese Society of Nephrology: eGFR $\left(\mathrm{ml}^{\prime} \mathrm{min}^{-1} \cdot 1.73 \mathrm{~m}^{-2}\right)=194 \times \mathrm{Serum}_{\mathrm{creatinine}} \mathrm{e}^{-}$ $1.094 \times \mathrm{Age}^{\wedge}-0.287$ (if female: $\times 0.739$ ). Average IMT was the mean value of 60 computer-based points in the $20 \mathrm{~mm}$ proximal region to the origin of the carotid bulb.

${ }^{*}$ Change from baseline in each group was assessed using paired t-test. ${ }^{+}$Changes between the 2 treatment groups were assessed using analysis of covariance, including treatment groups as the main effect and baseline variable as covariate. Log-transformed values were used in statistical tests of BNP and urinary albumin.

IMT, intima-media thickness; eGFR, estimated glomerular filtration rate; ND, not determined; BNP, B-type natriuretic peptide. Other abbreviations as in Table 3.

changes in morning HBP between the 2 groups with $80 \%$ power at 2-tailed 5\% significant level. Data analyses were carried out on the full analysis set. Changes in blood pressure and other parameters between the 2 groups were compared using analysis of covariance, including treatment groups as main effect and baseline value as covariate. The achievement rates of the target blood pressure levels were compared using the Fisher's exact test. All analyses were performed using SAS statistical software (version 9.2; SAS Institute Inc, Cary, NC, USA).

\section{Results}

\section{Patient Characteristics}

A total of 316 patients were registered from September 2004 to February 2006. Fifty-three patients were excluded as a result of failure to meet the inclusion criteria, consent withdrawal, lost to follow-up, or miscellaneous reasons during the observation period. Thus, 263 patients were randomly allocated to the increased ARB group $(n=132)$ and the combination group $(n=131)$, all cases for analysis (Figure 1). Baseline patient characteristics were similar between the 2 groups (Table 1 ), except for the duration of diabetes which was longer in the combination group $(\mathrm{P}=0.014)$. In the increased ARB group, more patients received antihypertensive drugs other than the study drugs additionally throughout the 1-year study period (Table 2). Anti-diabetic agents were used comparably in the both groups.

\section{Changes in Blood Pressure}

For the primary endpoint, the combination group had significantly lower morning HBP than the increased ARB group at
1 year $(\mathrm{P}<0.001$ for systolic, $\mathrm{P}=0.010$ for diastolic, Table 3$)$, although more patients refractory to the study drugs received antihypertensive drugs additionally in the increased ARB group than the combination group (Table 2). At 8 weeks, the increased ARB group showed almost no change of morning HBP, whereas the combination group had lower morning HBP (intergroup comparison: $\mathrm{P}<0.001$ for systolic and diastolic, Table 3 ). The combination group showed greater antihypertensive effect than the increased ARB group in evening HBP and office blood pressure during the study period (Table 3 ). We confirmed that imbalance of the duration of diabetes at baseline did not affect the results with analysis adjusting for the duration of diabetes. The combination group produced significantly higher achievement rates of target blood pressure level than the increased ARB group at 8 weeks (Figure 2). The achievement rates of the morning HBP were less than $10 \%$ in the 2 groups at 1 year.

\section{Changes in Biomarkers and Other Parameters}

$\mathrm{BNP}$ increased at 1 year in the increased $\mathrm{ARB}$ group $(\mathrm{P}=0.019)$, whereas that in the combination ARB group did not, showing significant difference between the 2 groups ( $\mathrm{P}=0.049$, Table 4). Urinary albumin levels did not change significantly in both groups at 1 year. However, the reduction of urinary albumin was positively correlated with the reduction of HBP than that of office blood pressure at 1 year (Table 5). Estimated glomerular filtration rate (eGFR) decreased significantly from baseline in the increased ARB group at 1 year $(\mathrm{P}=0.005)$, while that in the combination group decreased but not significantly. The average IMT increase in the increased group from baseline, while that in the combination group decrease, showing tendency 


\begin{tabular}{|c|c|c|c|c|}
\hline & \multicolumn{2}{|c|}{ Increased ARB group } & \multicolumn{2}{|c|}{ Combination group } \\
\hline & $\mathbf{r}$ & $P$ value & $\mathbf{r}$ & $P$ value \\
\hline$\Delta$ Morning SBP vs. $\triangle$ UACR & 0.248 & 0.034 & 0.406 & $<0.001$ \\
\hline$\Delta$ Morning DBP vs. $\triangle$ UACR & 0.258 & 0.027 & 0.360 & 0.001 \\
\hline$\Delta$ Office SBP vs. $\Delta$ UACR & 0.088 & 0.404 & 0.266 & 0.012 \\
\hline$\Delta$ Office DBP vs. $\triangle$ UACR & 0.080 & 0.447 & 0.156 & 0.146 \\
\hline At 1 year, Morning SBP vs. UACR & 0.089 & 0.456 & 0.212 & 0.066 \\
\hline At 1 year, Morning DBP vs. UACR & -0.036 & 0.764 & -0.107 & 0.359 \\
\hline
\end{tabular}

$\triangle$, [at 1 year]-[at baseline]; r, Pearson's correlation coefficient with log-transformed UACR.

UACR, urinary albumin. Other abbreviations as in Table 3.

of significance between the 2 groups at 1 year $(\mathrm{P}=0.073)$.

\begin{abstract}
Safety
Adverse events were reported in 45 patients $(34.1 \%)$ of the increased ARB group and in 51 patients $(38.9 \%)$ of the combination group. The study termination as a result of adverse events occurred in 6 patients $(4.5 \%)$ and 4 patients $(3.1 \%)$, respectively. Dizziness was observed in 4 patients in each group. Edema was found in 2 patients of the increased ARB group and in 3 patients of the combination group. Postural hypotension was found in 3 patients of the increased ARB group. Hepatic dysfunction was found in 7 patients in each group. Elevations of serum creatinine were observed more frequently in the combination group ( 1 patient vs. 7 patients). None of these events had any adverse clinical consequence. Other adverse events were observed comparably between the 2 groups, and each treatment was well tolerated.
\end{abstract}

\section{Discussion}

In these initial 8-week and 1-year data from the ADVANCED$\mathrm{J}$ study, we found that the amlodipine combined with ARB provided significantly greater antihypertensive effect than increased dose of ARB during the study period in type 2 diabetic patients with hypertension. Furthermore, our trial adopted self-measured HBP using an automated sphygmomanometer and an automated data transfer system to obtain more accurate blood pressure measurements as endpoints. To our knowledge, this is the first study to demonstrate the efficacy of combination therapy of antihypertensive drugs on HBP for relatively long-term period in diabetic patients with hypertension.

Blood pressure control in diabetic patients remains a considerable clinical important issue. In particular, these patients are at high risk of cardiovascular events, such as myocardial infarction and stroke. ${ }^{1,11}$ Several large clinical studies demonstrated that adequate control of hypertension in diabetic patients decreased the risk of cardiovascular events. ${ }^{4-6}$ Furthermore, a previous study demonstrated that each $10 \mathrm{mmHg}$ increase in home systolic blood pressure increased the risk of cardiovascular events by $17.2 \% .^{12}$ The present study showed that the differences in morning systolic blood pressure between the 2 groups were $12 \mathrm{mmHg}$ at 8 weeks and $10 \mathrm{mmHg}$ at 1 year. Our results suggest that significant reduction of HBP with combination of amlodipine and ARB anticipates the possibility of decreasing incidence of cardiovascular events in the future. Advantage of combination therapy by ACCOMPLISH trial ${ }^{13}$ showed that angiotensin II converting enzyme inhibitor (ACEI) and calcium channel blocker (CCB) combination therapy was superior to ACEI and diuretic in clinical outcomes.

The achievement rate of target blood pressure level of am- lodipine combination therapy was still low in the present study. Most of the participants were treated with only single ARB before the study treatment, although systolic blood pressure was comparatively high. More than half of the participants were aged 65 years and over. Their characteristics might be considered as the factors for not achieving the target blood pressure recommended by the guideline, although other anti-hypertensive drugs were available after 8 weeks. Our result reflected the actual situation of routine care and suggested that it was difficult to control hypertension adequately in diabetic patients with $\mathrm{CCB}$ and standard dose of ARB. Clinical evidence also shows that achieving target blood pressure level requires an average of 3.2 different antihypertensive drugs. ${ }^{14}$

The reduction of blood pressure in the combination therapy was observed over the short period of 8 weeks, indicating that this therapy provides the clinically beneficial effect. These findings are supported by the VALUE study ${ }^{15}$ and the ASCOTBPLA study, ${ }^{16}$ which demonstrated that early normalization of blood pressure is important in sustaining a long-term better control of blood pressure and reducing cardiovascular events in patients with hypertension who are at high risk of cardiovascular events. The difference of blood pressure between the 2 groups did not become small during the study period, even though all antihypertensive drugs were available through the study period, suggesting that it is not easy to control hypertension without $\mathrm{CCB}$ and $\mathrm{ARB}$ in diabetic patients, although only they are not enough to control hypertension.

Several studies had reported that self-measurement of HBP was useful for detection of insufficient control of blood pressure ${ }^{17}$ and it had better prognostic accuracy in terms of both cardiovascular morbidity and mortality than the office measurement. ${ }^{12,18}$ HBP measurements have also been shown to predict the damage of target organ by hypertension better than office measurements. ${ }^{8}$ In fact, our study showed that the reduction of urinary albumin was positively correlated with the reduction of HBP than that of office blood pressure. Of note, we observed the sustained antihypertensive efficacy of combination therapy in which reduction of systolic blood pressure from office measurement was sustained in the evening and the elevation of morning HBP was less in contrast to the increased ARB therapy during the study period. These findings indicate that HBP monitoring and amlodipine combination therapy with $\mathrm{ARB}$ should be used in clinical practice, and might provide better prognosis in diabetic patients with hypertension.

The BNP level provides predictive information for future onset of cardiovascular disease. ${ }^{19}$ Our study showed that BNP increased significantly in the increased $A R B$ regimen at 1 year, whereas that in the combination regimen did not change, showing the significant difference in the 2 groups. The positive correlation between blood pressure and BNP is well known. ${ }^{20}$ Our 
study did not show the positive correlation of BNP reduction and the reduction of HBP. However, because BNP value and home systolic blood pressure were correlated positively in both groups at 1 year $(\mathrm{r}=0.271, \mathrm{P}=0.017$ for the increased $\mathrm{ARB}$ regimen; $r=0.350, P=0.002$ for the combination regimen), it was suggested that more potent reduction of HBP might be preferable to avoid the exacerbation of BNP.

The sub-analysis of NICE-Combi study showed that nifedipine and standard dose of candesartan combination regimen reduced urinary albumin excretion than maximal dose of candesartan in hypertensive patients with miciroalbuminuria with the 8-week treatment. ${ }^{21}$ Our study did not obtain the superiority of combination regimen to increased ARB regimen in reduction of urinary albumin. As one of this reason, it might be considered that urinary albumin level was low; half of patients were categorized into normoalbuminuria in contrast to the NICE-Combi Study. However, it was suggested that adequate management of HBP is important to reduce urinary albumin as a result of their correlation from our results.

In addition, at 1 year, eGFR decreased significantly from baseline in the increased ARB regimen, whereas that in the combination therapy decreased but not significantly. A previous study showed reduced eGFR was independent risk factors for cardiovascular events and mortality rates in patients with diabetes. ${ }^{22}$ Risk of cardiovascular events was further modified by renal status changes over the first 2 years. End-stage renal disease was too late to improve the outcomes. Therefore, early intervention in early stage was an important strategy for prevention of adverse events. From this point of view, combination therapy at least retarded the progression of renal dysfunction. Furthermore, carotid IMT in the combination group tends to be less than that in ARB group at 1 year. O'Leary et al demonstrated that increases in the thickness of the carotid IMT were directly associated with an increased risk of myocardial infarction and stroke. ${ }^{23}$ The estimated cumulative rate of the combined end point for the highest quintile of the combined wall-thickness measure was over 25 percent at 7 years, as compared with a cumulative rate of less than 5 percent for the lowest quintile. These surrogate endpoints also suggested that the combination therapy is more preferable than the increased ARB therapy in the management for hypertension in diabetic patients.

Several limitations of the study warrant mention. First, it was conducted as an open label design. Second, the patients might have become more health-conscious during the course of the study or undertaken other measures that might have influenced the results. However, any such change was likely to have affected both groups equally, and was therefore unlikely to have influenced the results. Third, follow-up data was not available for some patients. However, we also confirmed the efficacy in main analyses with the last observation carried forward analysis. Therefore, we judged the missing data did not affect our overall conclusions.

In summary, these 1-year data from the ADVANCE-J Study indicate that amlodipine combination therapy with ARB produces significant lowering in blood pressure in diabetic patients with hypertension refractory to standard dose of ARB. These findings have significant implications for the care of patients with high risks of cardiovascular events.

\section{Acknowledgment}

We thank the participating research institutions and investigators as follows: Fumio Murayama, International University of Health and Welfare Hospital; Takeshi Osonoi, Nakakinen Clinic; Katsumi Miyauchi and Hirotaka Watada, Juntendo University Hospital; Kazuhide Yamaoki, Sanno Hospital;
Atsuhisa Sato, International University of Health and Welfare Mita Hospital; Eiji Tamiya, Koto Hospital; Takeshi Kurata, Kurata Clinic; Dobun Hayashi, Ochanomizu Hijiribashi Clinic; Tsutomu Yamazaki, The University of Tokyo Hospital; Fuki Ikeda, Chiba Tokusyukai Hospital; Kosei Tanimoto, Juntendo University Urayasu Hospital; Akio Kanazawa, International Goodwill Hospital; Shunichi Tanaka, Kanazawa Medical Clinic; Yoshihiko Yamada and Tomohiko Shigemasa, International University of Health and Welfare Atami Hospital; Jong Bock Choi and Satoru Suwa, Juntendo University Shizuoka Hospital; Issei Shimada, Shimada General Hospital; Masao Ohashi, Fukuoka Sanno Hospital; Yasuhiro Ono, Takagi Hospital; Shiro Hata, National Hospital Organization Ureshino Medical Center.

\section{Disclosures}

Authors report receiving research grants as follows: K.M., from Astellas Pharma Inc, Daiichi Sankyo Co, Ltd, Dainippon Sumitomo Pharma Co Ltd, MSD K.K., Novartis Pharma K.K., Pfizer Japan Inc and Takeda Pharmaceutical Co, Ltd; T.Y., from DSP, MSD, NP, PJ, and TP; H.W., from AP, Boehringer Ingelheim Japan, Inc, DS, DSP, MSD, NP, PJ, and TP; Y.T., from BIJ, DS, DSP, MSD, NP, PJ, and TP; R.K., from AP, BIJ, DS, DSP, MSD, NP, PJ, and TP; Y.I., from AP, BIJ, DS, DSP, MSD, NP, PJ, and TP; S.I., from AP, PJ, and TP; S.S., from AP, BIJ, DS, DSP, MSD, NP, PJ, and TP; H.D., from AP, BIJ, DS, DSP, MSD, NP, PJ, and TP. The remaining authors report no conflicts.

Source of Funding: The ADVANCED-J study was supported by the Japan Heart Foundation.

Conflict of Interest: All authors have declared any relevant financial relationships in Authorship Agreement.

Name of grant: The Japan Heart Foundation.

\section{References}

1. Sowers JR, Epstein M, Frohlich ED. Diabetes, hypertension, and cardiovascular disease: An update. Hypertension 2001; 37: 1053-1059.

2. Asman G, Cullen P, Schulte H. Simple scoring scheme for calculating the risk of acute coronary events based on the 10-year follow-up of the Prospective Cardiovascular Münster (PROCAM) study. Circulation 2002; 105: 310-315.

3. Hansson L, Zanchetti A, Carruthers SG, Dahlöf B, Elmfeldt D, Julius $\mathrm{S}$, et al and for the HOT Study Group. Effects of intensive bloodpressure lowering and low-dose aspirin in patients with hypertension: Principal results of the Hypertension Optimal Treatment (HOT) randomised trial. Lancet 1998; 351: 1755-1762.

4. Tuomilehto J, Rastenyte D, Birkenhäger WH, Thijs L, Antikainen R, Bulpitt CJ, et al; for The Systolic Hypertension in Europe Trial Investigators. Effects of calcium-channel blockade in older patients with diabetes and systolic hypertension. N Engl J Med 1999; 340: 677684 .

5. Bakris GL, Williams M, Dworkin L, Elliott WJ, Epstein M, Toto R, et al; for the National Kidney Foundation Hypertension and Diabetes Executive Committees Working Group. Preserving renal function in adults with hypertension and diabetes: A consensus approach. Am J Kidney Dis 2000; 36: 646-661.

6. McFarlane SI, Jacober SJ, Winer N, Kaur J, Castro JP, Wui MA, et al. Control of cardiovascular risk factors in patients with diabetes and hypertension at urban academic medical centers. Diabetes Care 2002; 25: 718-723.

7. Pickering TG, Shimbo D, Haas D. Ambulatory blood-pressure monitoring. N Engl J Med 2006; 354: 2368-2374.

8. Mallick S, Kanthety R, Rahman M. Home blood pressure monitoring in clinical practice: A review. Am J Med 2009; 122: 803-810.

9. Brooks CS, Sowers JR. Importance of achieving lower blood pressure in hypertensive patients with diabetes. Hypertension 2004; 44: $614-615$.

10. Kawamori R, Daida H, Tanaka Y, Miyauchi K, Kitagawa A, Hayashi $\mathrm{D}$, et al. Amlodipine versus angiotensin II receptor blocker; control of blood pressure evaluation trial in diabetics (ADVANCED-J). BMC Cardiovasc Disord 2006; 6: 39-44.

11. Ueshima K, Oba K, Yasuno S, Fujimoto A, Tanaka S, Ogihara T, et al. Influence of coronary risk factors on coronary events in Japanese high-risk hypertensive patients: Primary and secondary prevention of ischemic heart disease in a subanalysis of the Candesartan Antihypertensive Survival Evaluation in Japan (CASE-J) trial. Circ J 2011; 75: 2411-2416.

12. Bobrie G, Chatellier G, Genes N, Clerson P, Vaur L, Vaisse B, et al. Cardiovascular prognosis of "masked hypertension" detected by blood pressure self-measurement in elderly treated hypertensive patients. JAMA 2004; 29: 1342-1349.

13. Jamerson K, Weber MA, Bakris GL, Dahlöf B, Pitt B, Shi V, et al; for the ACCOMPLISH Trial Investigators. Benazepril plus amlodip- 
ine or hydrochlorothiazide for hypertension in high-risk patients. $N$ Engl J Med 2008; 359: 2417-2428.

14. Bakris GL, Williams M, Dworkin L, Elliott WJ, Epstein M, Toto R, et al. Preserving renal function in adults with hypertension and diabetes: A consensus approach: National Kidney Foundation Hypertension and Diabetes Executive Committees Working Group. Am J Kidney Dis 2000; 36: 646-661.

15. Julius S, Kjeldsen SE, Weber M, Brunner HR, Ekman S, Hansson L, et al; for the VALUE trial group. Outcomes in hypertensive patients at high cardiovascular risk treated with regimens based on valsartan or amlodipine: The VALUE randomised trial. Lancet 2004; 363: 2022 2031.

16. Poulter NR, Wedel H, Dahlöf B, Sever PS, Beevers DG, Caulfield M, et al; ASCOT Investigators. Role of blood pressure and other variables in the differential cardiovascular event rates noted in the AngloScandinavian Cardiac Outcomes Trial-Blood Pressure Lowering Arm (ASCOT-BPLA). Lancet 2005; 366: 907-913.

17. Park SJ, Park JB, Choi DJ, Youn HJ, Park CG, Ahn YK, et al. Detection of masked hypertension and the 'mask effect' in patients with well-controlled office blood pressure. Circ J 2011; 75: 357-365.

18. Niiranen TJ, Hänninen MR, Johansson J, Reunanen A, Jula AM. Home-measured blood pressure is a stronger predictor of cardiovascular risk than office blood pressure: The Finn-Home study. Hyper- tension 2010; 55: 1346-1351.

19. Sakuma M, Nakamura M, Tanaka F, Onoda T, Itai K, Tanno K, et al. Plasma B-type natriuretic peptide level and cardiovascular events in chronic kidney disease in a community-based population. Circ J 2010; 74: 792-797.

20. Corti R, Burnett JC Jr, Rouleau JL, Ruschitzka F, Lüscher TF. Vasopeptidase inhibitors: A new therapeutic concept in cardiovascular disease? Circulation 2001; 104: 1856-1862.

21. Nakagawa N, Fujino T, Kabara M, Matsuki M, Chinda J, Kikuchi K, et al; NICE-Combi Study Group. Angiotensin II receptor blocker and long-acting calcium channel blocker combination therapy decreases urinary albumin excretion while maintaining glomerular filtration rate. Hypertens Res 2011; 34: 1121-1126.

22. Hemmelgarn BR, Manns BJ, Lloyd A, James MT, Klarenbach S, Quinn RR, et al; Alberta Kidney Disease Network. Relation between kidney function, proteinuria, and adverse outcomes. JAMA 2010; 303: $423-429$.

23. O'Leary DH, Polak JF, Kronmal RA, Manolio TA, Burke GL, Wolfson SK Jr. Carotid-artery intima and media thickness as a risk factor for myocardial infarction and stroke in older adults: Cardiovascular Health Study Collaborative Research Group. N Engl J Med 1999; 340: $14-22$. 